PROCEEDINGS OF THE

AMERICAN MATHEMATICAL SOCIETY

Volume 104, Number 3, November 1988

\title{
METACYCLIC $p$-ALGEBRAS
}

\author{
MING-CHANG KANG
}

(Communicated by Donald Passman)

\begin{abstract}
Let $K$ be a field of $\operatorname{char} K=p>0, m, r$ any positive integers with $(p, m)=1$, and $L$ a metacyclic extension of $K$ with degree $p^{r} m$, i.e. $\operatorname{Gal}(L / K)=\left\langle\sigma, \tau: \sigma^{p^{r}}=\tau^{m}=1, \tau \sigma \tau^{-1}=\sigma^{e}\right\rangle$ for some integer $e$. If $A$ is a central simple $K$-algebra of degree $p^{r}$ and is split by $L$, then $A$ is a cyclic algebra. For $r=1$, the theorem has been proved by A. A. Albert [1].
\end{abstract}

1. Introduction. In [1], A. A. Albert proves the following theorem,

THEOREM. Let $K$ be any field of char $K=p>0, m$ any positive integer which is relatively prime to $p$, and $L$ a metacyclic extension of $K$ with degree $p m$, i.e. $\operatorname{Gal}(L / K)=\left\langle\sigma, \tau: \sigma^{p}=\tau^{m}=1, \tau \sigma \tau^{-1}=\sigma^{e}\right\rangle$ for some integer $e$. If $A$ is a central simple K-algebra of degree $p$ and is split by $L$, then $A$ is a cyclic algebra.

The aim of this note is to generalize the above theorem to the case of $p$-algebras of degree $p^{r}$. Namely,

THEOREM. Let $K$ be any field of char $K=p>0, m, r$ any positive integer with $(p, m)=1$, and $L$ a metacyclic extension of $K$ with degree $p^{r} m$, i.e. $\operatorname{Gal}(L / K)=$ $\left\langle\sigma, \tau: \sigma^{p^{r}}=\tau^{m}=1, \tau \sigma \tau^{-1}=\sigma^{e}\right\rangle$ for some integer $e$. If $A$ is a central simple $K$-algebra of degree $p^{r}$ and is split by $L$, then $A$ is a cyclic algebra.

\section{The proof of the theorem.}

LEMMA. Let $K$ be any field, $m, n$ any positive integers which are relatively prime, and $L$ a metacyclic extension of $K$ with degree $m n$, i.e. $\operatorname{Gal}(L / K)=$ $\left\langle\sigma, \tau: \sigma^{n}=\tau^{m}=1, \tau \sigma \tau^{-1}=\sigma^{e}\right\rangle$ for some integer $e$. If $A$ is a central simple $K$-algebra of degree $n$ which is split by $L$ and $F=\{a \in L: \sigma(a)=a\}$, then $A \otimes_{K} F$ is isomorphic to the cyclic algebra $(b, L / F, \sigma)$ for some $b \in F^{\times}$with $\tau(b)=\lambda^{n} b^{e}$ where $\lambda$ is some element in $F^{\times}$.

PrOOF. Let $C$ be a central simple $K$-algebra of degree $m n$ which is similar to $A$. Then $L$ is a maximal subfield of $C$. Let the centralizer of $F$ in $C$ be $B$. Then $B \cong A \otimes_{K} F$. The automorphism $\tau^{i}$ on $L, 0 \leq i \leq m-1$, can be extended to an automorphism on $C$; again we call it $\tau^{i}$. Then $\tau^{i}(B)=B$.

Now $L$ is a maximal subfield of $B$. Hence $B=(b, L / F, \sigma)$ for some $b \in F^{\times}$ [2, p. 71]. Then $\tau(B)=\left(\tau(b), L / F, \sigma^{e}\right)$ and $\tau^{i}(B)=\left(\tau^{i}(b), L / F, \sigma^{e^{i}}\right)$. Let us denote the similarity classes of $B$ and $(b, L / F, \sigma)$ by $[B]$ and $[b, L / F, \sigma]$ respectively. Let $f$ be an integer with $e f=1(\bmod n)$. Then, by [2, Lemma 3, p. 72],

Received by the editors January 12, 1988.

1980 Mathematics Subject Classification (1985 Revision). Primary 16A39, 12E15.

Key words and phrases. Central simple algebra, cyclic algebra.

Partially supported by the National Science Council, Republic of China. 
$[B]=\left[\tau^{i}(B)\right]=\left[\tau^{i}(b), L / F, \sigma^{e^{i}}\right]=\left[\tau^{i}\left(b^{f^{i}}\right), L / F, \sigma^{e^{i} f^{i}}\right]=\left[\tau^{i}\left(b^{f^{i}}\right), L / F, \sigma\right]$. Therefore $m[B]=\sum_{0 \leq i \leq m-1}\left[\tau^{i}(B)\right]=[c, L / F, \sigma]$ where $c=\prod_{0 \leq i \leq m-1} \tau^{i}\left(b^{f^{i}}\right)$.

Now

$$
\frac{\tau(c)}{c^{e}}=\frac{b^{f^{m-1}}}{b^{e}} \prod_{1 \leq i \leq m-1} \tau^{i}\left(\frac{b^{f^{i-1}}}{b^{e f^{i}}}\right) \in F^{\times^{n}}
$$

since $e f=1(\bmod n)$.

Choose integer $m_{0}$ with $m m_{0}=1(\bmod n)$. Then $[B]=m_{0}[c, L / F, \sigma]=$ $\left[c^{m_{0}}, L / F, \sigma\right]$. Clearly $\tau\left(c^{m_{0}}\right)=\lambda^{n}\left(c^{m_{0}}\right)^{e}$ for some $\lambda \in F^{\times}$. This completes the proof of the Lemma.

Now the proof of the theorem.

Let $n=p^{r}$ in the above Lemma. We have $A \otimes_{K} F$ is isomorphic to $(b, L / F, \sigma)$ for some $b \in F^{\times}$with $\tau(b)=\lambda^{p^{r}} b^{e}$ where $\lambda \in F^{\times}$.

Note that the cyclic algebra $(b, L / F, \sigma)$ has a maximal subfield $F(v)$ with $v^{p^{r}}=b$ and $[F(v): F]=p^{r}$. (If the polynomial $X^{p^{r}}-b \in F[X]$ is not irreducible, apply [2, Lemma 8, p. 75] to reduce the degree of $A \otimes_{K} F$ and rework the Lemma.)

Claim. $F(v)$ is a normal extension of $K$. Since $F$ is a Galois extension of $K$, it suffices to show that all conjugates of $v$ over $K$ are contained in $F(v)$. Let $f(X)$ be the minimal polynomial of $b$ over $K$. Then $v$ is a root of $f\left(X^{p^{r}}\right)=0$. Hence, if $u$ is a conjugate of $v$ over $K$, then $u$ is also a root of $f\left(X^{p^{r}}\right)=0$. It follows that $u^{p^{r}}$ is some conjugate of $b$, i.e. $u^{p^{r}}=\tau^{i}(b)$ for some $i$. But $\tau^{i}(b)=\lambda_{i}^{p^{r}} b^{e^{i}}$ for some $\lambda_{i} \in F^{\times}$. Hence $u^{p^{r}}=\lambda_{i}^{p^{r}}\left(v^{e^{i}}\right)^{p^{r}}$. It follows that $u=\lambda_{i} v^{e^{i}} \in F(v)$.

Since $F(v)$ is normal over $K$, it follows that $F(v)=F \otimes_{K} E$ where $E$ is the maximal purely inseparable extension in $F(v)$. Clearly, the purely inseparable exponent of $F(v)$ over $F$ and that of $E$ over $K$ are the same, which is just $r$. Hence $E=K(w)$ for some $w$ with $w^{p^{r}} \in K$.

Consider $A \otimes_{K} K(w)$. Since $F(v)$ splits $A \otimes K(w)$ and $[F(v): K(w)]$ is relatively prime to the degree of $A \otimes_{K} K(w)$. It follows that $\left[A \otimes_{K} K(w)\right]=0$ in $\operatorname{Br}(K(w))$. By [2, Theorem 2, p. 108], $A$ is a cyclic algebra.

\section{REFERENCES}

1. A. A. Albert, A note on normal division algebras of prime degree, Bull. Amer. Math. Soc. 44 (1938), 649-652.

2. P. K. Draxl, Skew fields, London Math. Soc. Lecture Note Ser., Cambridge Univ. Press, Cambridge, 1983.

Department of Mathematics, National Taiwan University, Taipei, Taiwan REPUBLIC OF CHINA 